\title{
Programming ABB Industrial Robot for Writing a Human Language
}

\author{
Phongsavanh Sengaphone \\ Masters student, Dept. of Mechanical \\ Tianjin University of Technology and Education (TUTE), \\ Tianjin, China
}

\begin{abstract}
Using Robotics writes a human's language that is interesting by the researchers in the robotics field. This paper devoted to programming ABB Robot to write some characters Chinese language (机器人), and attach a special end effector instrument to the ABB Robot. The writing instrument had been designed and fabricated that become a tool (end effector). The tool that is helping ABB Robot can control the pen to write the letters is better. The RAPID program is uploaded as a backup system into the flex pendant of the robot and then simulated through the IRC5 controller. As a result, the project was able to simulate some Chinese characters, and then the character was written by the ABB Robot accurately.
\end{abstract}

Key-word- ABB industrial Robot, Robot Studio, Writing Instrument, RAPID.

\section{INTRODUCTION}

A robot is a human invention that establishes for replaces human effort albeit it may not resemble a human. A robot is designed and controlled by a computer or some equipment that be similar to computers. The system of the robots that can be used together with the robots to operate the essential works. The movement of the robot is controlled by a controller that is under the jurisdiction of the computer that is running some type of program. Therefore if the program is changed, the actions of the robot will be changed accordingly. Thus the robot is designed to be able to operate any work that can be programmed (within its limit and work envelope) simply by changing the program [1]

The target of this project is programming industrial Robot to work instead of a human, in this case, was a programmed robot for writing. This programming will use with industrial Robot (ABB IRB 120) by using RobotStudio software and RAPID (GUI) programming language. The IRB 120 ABB has a flex pendant that uses to control itself and control module to upload the command.

\section{SYSTEM DESCRIPTION}

To show this project, this is a block diagram of the system is shown in Fig. 1.

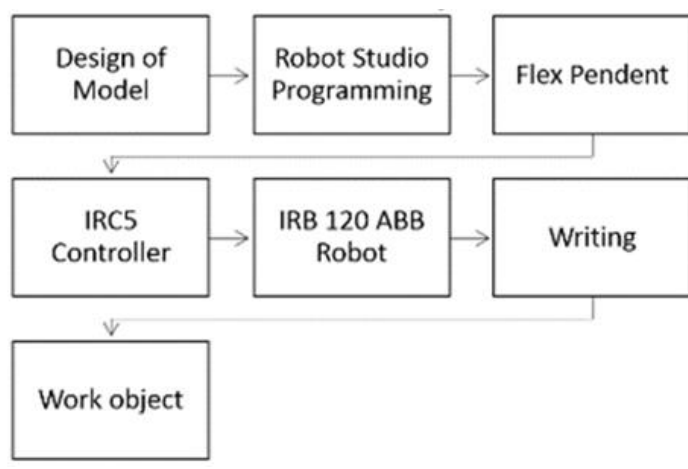

Fig. 1: System block diagram

\section{A. The SolidWorks Design Software}

The software is used in this project for the design of the end effector and the design of the alphabet letters that are SolidWorks Design software [2]. SolidWorks is a kind of software that can design a lot of solid models. Especially, that can create 3D solid models form graphical.

\section{B. RobotStudio Programming}

RobotStudio is a Graphical User Interface (GUI) software that uses configuration and offline programming of $\mathrm{ABB}$ robots with RAPID programming as the basic programming language. In the RobotStudio has a robot virtual technology that helps the User watch a robot work a RAPID program in the graphical environment, and can test the robot virtual that performs follow the paths and targets in programming are within the workspace of the robot manipulator or not [3] After write programming, a virtual controller that is also in RobotStudio, and then all of the paths are synchronized before off-line simulate. RobotStudio has an offline simulation tool that can be used to watch the motions of the robot while during simulation. Everything errors in the simulation that will also conduct to an error in the interfacing [4]. Through this off-line simulation, the program that can modify before uploading them into the flex pendant (Teach Pendant unit).

\section{Flex pendant}

The flex pendant which is also referred to as the teach pendant unit, is a hand held operator unit used to perform many of the tasks involved during the operation of a robot system such as modifying the work object, jogging the robot manipulator, modifying RAPID programs and can also be used to create instructions using teach method and jogging [5]. 


\section{IRC5 Controller}

The IRC5 controller contains all the functions required to move and control the robot manipulator. The controller consists of two modules, the control module and the drive module. The control module contains all the control electronics such as main computer I/O boards and flash memory. The control module runs all software necessary for operating the robot system. The drive module contains all the power electronics supplying the robot motors. An IRC5 drive module may contain nine drive units and can handle six internal axes plus two or additional axes depending on the robot model [6].

\section{E. IRB 120 Manipulator}

The IRB 120 is one of ABB Robotics latest generation of 6axis industrial robot, with a payload of $3 \mathrm{~kg}$, designed specifically for manufacturing industries that use flexible robot-based automation. The robot has an open structure that is especially adapted for flexible use, and can communicate extensively with external systems [7]. The IRB 120 robot manipulator has a weight of $25 \mathrm{~kg}$ and a repeatability position accuracy of $0.01 \mathrm{~mm}$. The average speed of the robot based on TCP velocity is $6.2 \mathrm{~m} / \mathrm{s}$ [8].

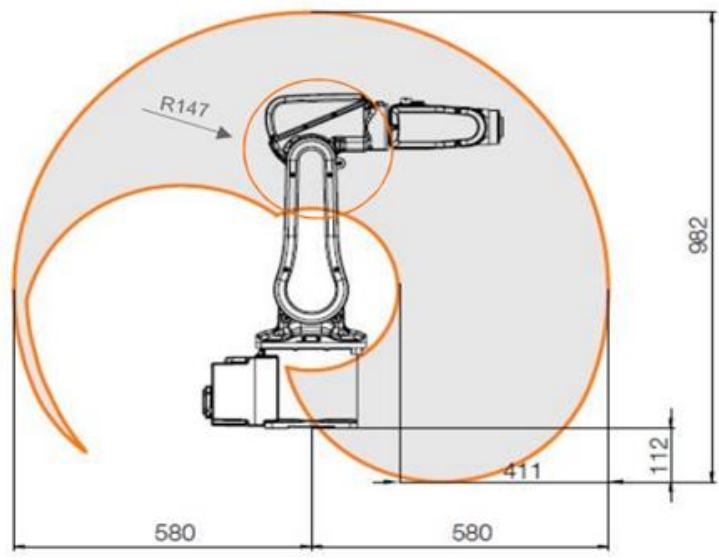

Fig. 2: Workspace Diagram of IRB 120 Robot

\section{F. End Effector for Writing}

The end effector is connected to the last joint of the manipulator (IRB 120). The end effector used in this project was writing that was designed with SolidWorks software for use in the program RobotStudio, and then attach to the ABB Robot of the program. The end effector as shown in Fig 3, the end effector was designed by SolidWorks software that designed separately and then come to combine, the first has dimension of wide $22 \mathrm{~mm}$, length $55 \mathrm{~mm}$, height $15 \mathrm{~mm}$ with a hole of $15 \mathrm{~mm}$ diameter for the piece of solid, and the last has dimension of wide 22 $\mathrm{mm}, 40 \mathrm{~mm}$, height $15 \mathrm{~mm}$ with a hole of $15 \mathrm{~mm}$ diameter for the pen. The length of the pen is about $80 \mathrm{~mm}$.

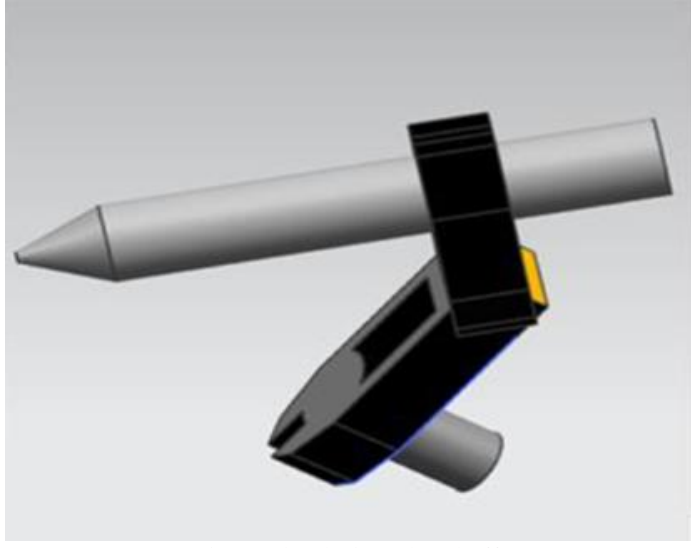

Fig 3: The designed end effector

\section{PROJECT DEVELOPMENT}

The writing tool was designed by SolidWorks software after the writing tool design finishes that be able to import in RobotStudio, which is created for use in the RAPID programming. After the tool (end effector) is created, a writing workstation is created in RobotStudio where the position of the work object in RobotStudio is set within the robot's workspace. The SolidWorks design of the letters that create a structure like a modelled box, the measured data of the model, inclusive height $120 \mathrm{~mm}$ (font height 5 $\mathrm{mm}$, base height $115 \mathrm{~mm}$ ), width $210 \mathrm{~mm}$, and length 297 $\mathrm{mm}$. after that imported into the RobotStudio and positioned on the writing work object at the same position initially set The letters are using the ordered (border around the surface) and created around the letters that have the letters on the modeled box of a surface. Next, path and targets are then created from the modeled letters and the command for the paths are changed to RAPID programming language. The modelled box as shown in Fig 4.page.

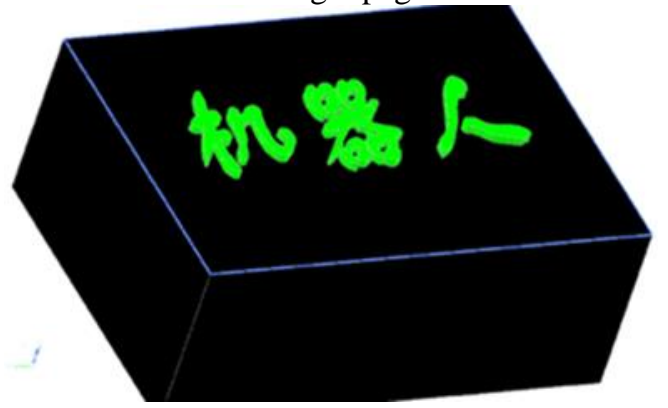

Fig. 4: The designed a modelled box

After finish programming, that is uploaded into the flex pendant and then the commands are uploaded in the controller. The controller can control the motion of the speed robot and coordinates the motions with the paths and targets. All the routines and paths of the writing instructions are executed by the robot on the work object after writing. The programming flowchart is shown in Fig. 5. 


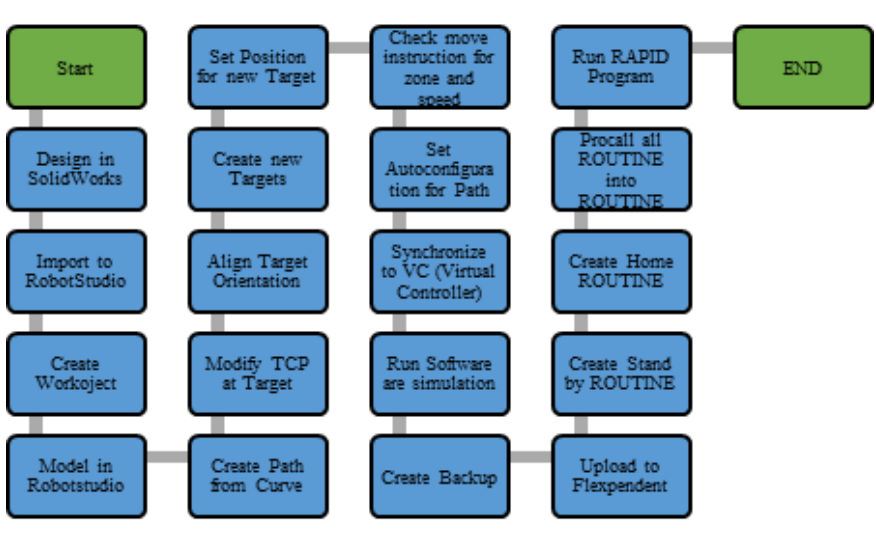

Fig. 5: Flow chart for software programming

A. Importing the End Effector into RobotStudio

After finish the design in SolidWorks, and then saved in *.sat or *ACIS format because both formats can be used in RobotStudio software. The design tool (end effector) is imported in RobotStudio and then setting tool to be aligning it with the origin in RobotStudio, and then attached it to the ABB RobotStudio. Additionally, that can save this tool in the RobotStudio (library file) which is convenient when want to change the tool.

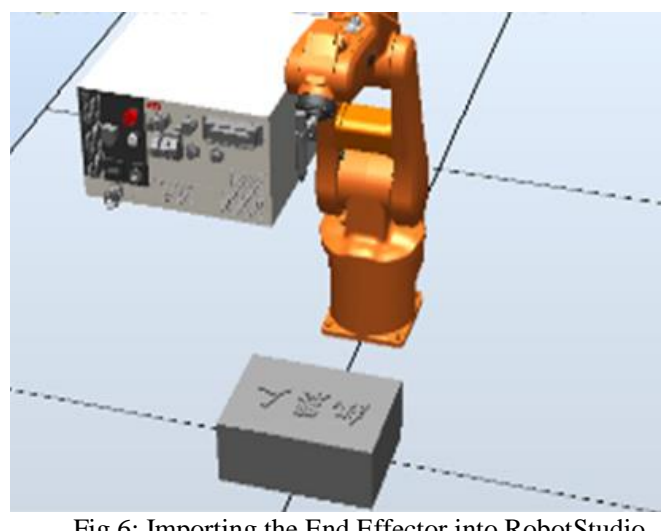

Fig 6: Importing the End Effector into RobotStudio

\section{B. Programming the Letter}

For the writing routine, targets were created a lot of point letter by following model design the path to be rapid. After the path programming, all the parts are then synchronized into the virtual controller in RobotStudio [9]. The results are shown in Fig.7.

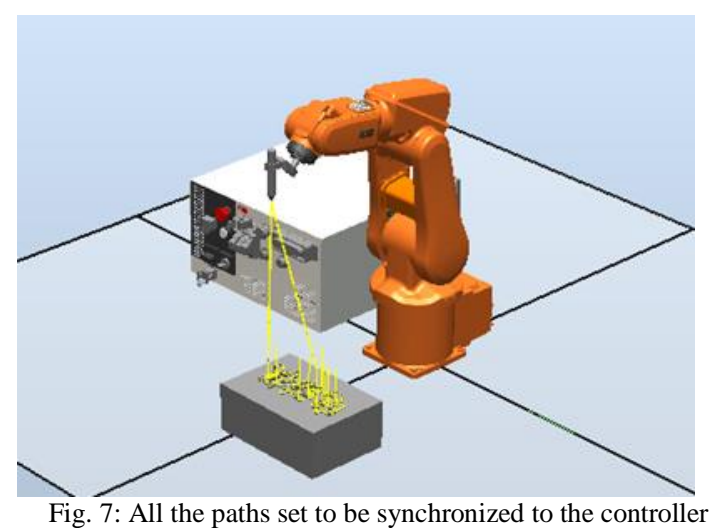

\section{RESULTS AND DISCUSSIONS}

\section{A. Result}

Figs. 8-9 show the picture of the end effector attach the $\mathrm{ABB}$ Robot, and the written letters on the paper routine.

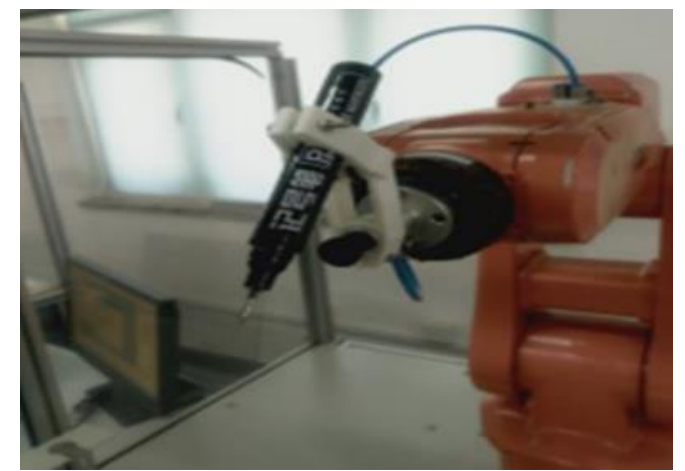

Fig 8: Results from the end effector attach the ABB Robot

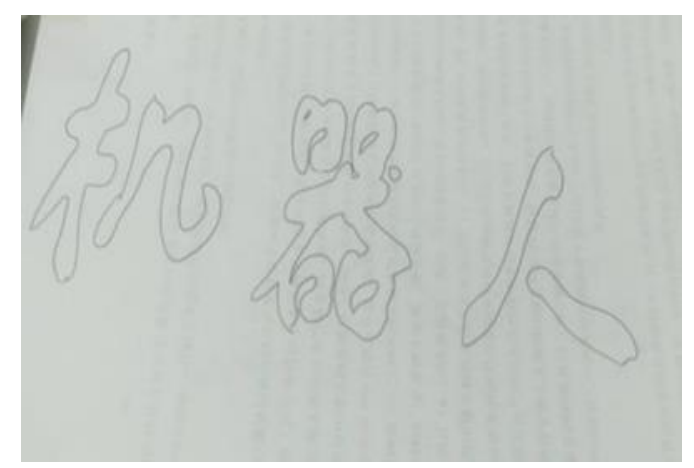

Fig 9: Results from robot interfacing for the letters

\section{B. Discussion}

The programming will simulation in the RobotStudio before that uploaded into the Flex pendant, the RobotStudio GUI simulation is the same as the interfacing, and the virtual Robot shows the actual simulation of the robot moving through the paths and targets. The program was uploaded into the flex pendant as a backup file and then restored into the system. The work object was adjusted to enable the writing tool to reach the work object. In the first experiment, the robot writing was a mistake because of the set position work object in RobotStudio and the work object used in the interfacing had a different position, that makes work object and end effector can't touch. This problem was solved by attaching equipment to help up and down at writing tool (paper), and then the robot can work follow the program all points. In the flex pendant, a new routine was created using the teaching method to jog the robot to two positions, the standby position and the home position. All the 3 letters of the alphabet were successfully programmed and both the offline and Robot Interfacing were successful. Using the same methodology, I was able to design other models of different languages by SolidWorks software, and then import them into RobotStudio for using $\mathrm{ABB}$ robot writing another language. 


\section{CONCLUSION}

In this paper, the programming $\mathrm{ABB}$ industrial robot for writing a human language, that is successful. In addition, still using SolidWorks software to design a tool (end effector) for the robot accurate writing. The Robot was able to write with the maximum speed and accuracy for all paths and routines created from the letters.

\section{REFERENCES}

[1] Saeed B. Niku, Ph.D., P.E Introduction to Robotics Analysis, Systems and Applications San Luis Obispo, California, Prentice Hall 2001, pp. 4-26, pp. 29-87, pp. 95-116.

[2] Solidworks, SolidWorks Essentials Training. 2008. Pp 1 -250.

[3] ABB Robotics RobotStudio courseware 5.11, 2008. Pp. 1-81.

[4] ABB Robotics, Operating Manual RobotStudio 5.13, 2010. Pp 91.

[5] ABB Robotics, Trouble shooting manual for IRC5 Controller, 2010. Pp $79-283$.

[6] ABB Robotics IRC5 Training Manual, 2011. Pp. 1-190.

[7] Product specification IRB 120, Pp 9.

[8] ABB's smallest robot - IRB 120 for flexible \& compact production.

[9] Mehmet Ismet Can Dede, and Sabri Tosunoglu, "Virtual RAPID Robot Prototyping" ASME Early Career Technical Journal, October 2006, Florida International University Miami, Florida. Pp. 1-5. 